\title{
Growth and Coagulation in a Herding Model
}

\author{
S. Rawal and G. J. Rodgers * \\ Department of Mathematical Sciences, Brunel University, \\ Uxbridge, Middlesex UB8 ЗPH, U.K.
}

\begin{abstract}
We discuss various existing models which mimic the herding effect in financial markets and introduce a new model of herding which incorporates both growth and coagulation. In this model, at each time step either (i) with probability $p$ the system grows through the introduction of a new agent or (ii) with probability $q=1-p$ two groups are selected at random and coagulate. We show that the size distribution of these groups has a power law tail with an exponential cut-off. A variant of our basic model is also discussed where rates are proportional to the size of a group.

PACS: 02.50.cw; 05.40.-a; 89.75.Hc

Keywords: Herding, growth, coagulation, power-laws
\end{abstract}

${ }^{*}$ Corresponding author. Tel.: +44-1895-203202; fax: +44-1895-203303.

E-mail address: G.J.Rodgers@brunel.ac.uk (G.J.Rodgers). 


\section{INTRODUCTION}

From the empirical analysis of financial price-data on short time scales, it appears that the price variations of many assets, indices and currencies deviate from the Gaussian distribution and have fat-tails [1-3]. Exceptionally large fluctuations are distributed according to an exponentially truncated power law [4] and it is believed that this behaviour is brought about by a herding effect.

The term herding can be described as a group of agents sharing the same information or the same rumour and imitating each other when making trading decisions $[5,6]$. These groups or herds of agents are thought to be responsible for the large fluctuations in prices. Herding coupled with supply and demand can cause sudden rises or falls when the groups all decide to buy/sell. The large groups have a strong impact on the supply and demand equilibrium, hence the larger the group, the bigger the impact they have on the market.

Several models have been proposed recently to mimic and investigate the herding behaviour in financial markets [7-13]. In these models, a cluster is a connected group of agents who can exchange information and make the same decisions from a choice of buying, selling or doing nothing. If we assume that the traded amount is proportional to the number of agents in a cluster [8], then the cluster size distribution can correspond to the distribution of returns i.e. the relative difference between the number of buyers and the number of sellers, or alternatively, the supply and demand balance [14], at a particular time.

Cont and Bouchaud [7] introduced a model in which the network of agents was considered to be a diluted regular lattice where the vertices were agents and edges were channels of communication. In this model, any agents were connected with probability $p$ and agents in a connected cluster formed a group which shared information and made the same decisions. A power law distribution with an exponential cut-off of group sizes, which corresponds to a distribution of returns, was obtained when the parameter $p$ was close to the percolation threshold.

To further investigate the herding effect, Eguíluz and Zimmermann [8] put forward a 
kinetic version of the above model in which, instead of being static, the network of agents evolved dynamically as decisions were being made or as agents exchanged information. They obtained numerically the same power law distribution for returns as Cont and Bouchaud [7]. An exact analytical expression was found for this model in Ref. [11] and since then alternative kinetic models have been introduced [12,13]. These three models [11-13] are very closely related as they incorporate only two phenomena from either growth, fragmentation, coagulation, addition, or attachment; however it is the combination of these ingredients which cause the models to differ.

The model proposed in Ref. [11] is one where groups of agents can either coagulate or fragment at each time step. The model introduced in Ref. [12] is a combination of attachment and fragmentation and the third model [13] incorporates both growth and addition.

In this paper, we introduce an alternative kinetic model in which either the system grows through the introduction of a new agent or two groups of agents coagulate. In Section 2 we review the three existing kinetic models [11-13] and the main results obtained. In Section 3, we present our main model and show that the size distribution of groups of agents has a power law tail with an exponential cut-off. We assume that rates are independent of group sizes. In Section 4, we study a variant of the model in which groups are selected for coagulation with a rate proportional to group sizes. In this case large groups grow more quickly than small ones. This seems reasonable as it is more likely that two agents from larger groups would come into contact with each other. In the final section we discuss our work and draw some conclusions.

\section{EXISTING MODELS}

\section{Model A Fragmentation and Coagulation}

In the model proposed by D'Hulst and Rodgers [11], with probability $p$ a cluster is selected at random for fragmentation. That is, a group of agents trade, by using their shared information and the group is then broken up into individual agents. With a probability $1-p$ 
two clusters are selected at random and coagulated. This means that two groups decide to share information and form a new larger group. As a chemical process this can be written as $B_{r} \rightarrow r B_{1}$ with probability $p$ and $B_{r}+B_{s} \rightarrow B_{r+s}$ with probability $1-p$. All rates are proportional to group sizes.

This model was solved exactly and it was found that $n_{s}$ which is the number of clusters of size $s$, obeys

$$
n_{s}=\frac{(1-p)^{s-1}(2 s-2) !}{(2-p)^{2 s-1} s !^{2}} N_{0}
$$

where $N_{0}$ is the total number of agents. For large $s$, it was found that

$$
n_{s} \sim N_{0}\left(\frac{4(1-p)}{(2-p)^{2}}\right)^{s} s^{-5 / 2}
$$

which is power law with an exponent $5 / 2$ with an exponential cut-off that vanishes when $p=0$. This shows that the model is not self-organised as a power-law distribution for large transactions only appears when the parameter $p$ is tuned to a low value.

The variance of the distribution of returns, $\sigma^{2}$, which is given by

$$
\sigma^{2}=M_{3}-M_{2}^{2}=\frac{(1-p)(2-p)}{p^{3}}
$$

where

$$
M_{i} \equiv \frac{1}{N_{0}} \sum_{s=1}^{\infty} s^{i} n_{s}
$$

is the $i$ th moment of $n_{s}$, was obtained. The kurtosis was also computed, which indicates that there are heavy tails for every value of $p$. This shows that the model deviates from Gaussian behaviour.

\section{Model B Attachment and Fragmentation}

In the model introduced by Rodgers and Zheng [12], at each time step, with probability $p$ an incoming agent joins an existing cluster of size $k$ with a rate proportional to $k$ and with probability $1-p$ a cluster is selected at random and fragmented. As a chemical process this can be written as $B_{1}+B_{r} \rightarrow B_{r+1}$ with probability $p$ and $B_{r} \rightarrow r B_{1}$ with probability $1-p$. 
In this model, for large $t, n_{k}(t)$ which is the number of clusters with size $k$, is

$$
n_{k}(t)=\frac{p(1-p)}{\alpha} \Gamma(\beta) \frac{\Gamma(k)}{\Gamma(k+\beta)} t
$$

As $k \rightarrow \infty$,

$$
n_{k}(t) \sim t k^{-\beta}
$$

where

$$
\beta=2+\frac{1-p}{\alpha}=2\left[\frac{\sqrt{4 p /(1-p)+1}}{\sqrt{4 p /(1-p)+1}-1}\right] .
$$

Hence this model gives a power law distribution of groups of agents for all parameter values, with an exponent which can take any value greater than 2 .

\section{Model C Growth and Addition}

In the model presented by Rodgers and Yap [13], at each time step, with probability $p$ the system grows through the introduction of a new agent and with probability $1-p$ a free agent already in the system is added at random to a group of size $k$ with rate $k$. As a chemical process this can be written as $0 \rightarrow B_{1}$ with probability $p$ and $B_{r}+B_{1} \rightarrow B_{r+1}$ with probability $1-p$.

By using a similar method to that of the above model, it was found that when $p>1 / 2$

$$
n_{k}(t)=p(2 p-1) \Gamma\left(1+\frac{1}{1-p}\right) \frac{\Gamma(k)}{\Gamma\left(k+\frac{1}{1-p}\right)} t
$$

where as $k \rightarrow \infty$,

$$
n_{k}(t) \sim t k^{-\frac{1}{1-p}}
$$

In summary, exact analytical expressions were obtained for the above models. It was found that whilst one has a power law distribution for group sizes with a tuneable exponent, others have exponents that are determined by the model as universal exponents. 


\section{A MODEL WITH GROWTH AND COAGULATION}

We introduce a model in which at each time step one of the two events, chosen at random, can occur. With probability $p$ an agent joins the group but remains free. Alternatively, with probability $1-p$, two groups are selected for coagulation with a rate independent to group sizes. As a chemical process this can be written as $0 \rightarrow B_{1}$ with probability $p$ and $B_{r}+B_{s} \rightarrow B_{r+s}$ with probability $1-p$. Consequently the number $n_{s}(t)$ of clusters of size $s$ at time $t$ evolves like

$$
\frac{d n_{s}(t)}{d t}=\frac{q}{N(t)^{2}} \sum_{r=1}^{s-1} n_{r} n_{s-r}-\frac{2 q n_{s}}{N(t)}+p \delta_{s, 1}
$$

In this equation

$$
N(t)=\sum_{s=1}^{\infty} n_{s}(t)
$$

represents the number of groups and

$$
M(t)=\sum_{s=1}^{\infty} s n_{s}(t)
$$

is the number of agents in the system. The first term on the right-hand side of Eq. (10) describes the creation of a new cluster of size $s$ by coagulation of two clusters of size $r$ and $s-r$, with $r<s$. The second term describes the disappearance of a cluster of size $s$ by coagulation with another cluster and the final term represents the arrival of new agents in the system. Using rate equation (10) it is a simple matter to show that

$$
\frac{d N(t)}{d t}=2 p-1
$$

and

$$
\frac{d M(t)}{d t}=p
$$

Eq. (13) represents the fact that with probability $2 p-1$, on average, the number of groups increases by 1. Similarly, Eq. (14) indicates that with probability $p$ the number of agents increases by 1 . The form of Eqs. (10), (13) and (14) suggests that the solution for $n_{s}(t)$, for 
$s=1,2, \ldots$, is linear in time for large $t$ when $p>1 / 2$. In this limit we can solve Eqs. (13) and (14) to yield

$$
N(t)=(2 p-1) t \quad \text { and } \quad M(t)=p t .
$$

Writing

$$
n_{s}(t)=t c_{s}
$$

we find that

$$
c_{s}=\frac{1-p}{(2 p-1)^{2}} \sum_{r=1}^{s-1} c_{r} c_{s-r}-\frac{2(1-p) c_{s}}{2 p-1}+p \delta_{s, 1} .
$$

Introducing the generating function

$$
g(\omega)=\sum_{s=1}^{\infty} c_{s} \omega^{s}
$$

we find

$$
g(\omega)=\frac{2 p-1}{2(1-p)}[1-\sqrt{1-4(1-p) p \omega}],
$$

giving a size distribution for clusters of

$$
c_{s}=\frac{(2 p-1) p^{s}(1-p)^{s-1}(2 s-2) !}{s !(s-1) !} .
$$

Using Stirling's formula to expand this for large $s$, we have

$$
c_{s} \sim(4 p(1-p))^{s} s^{-3 / 2}
$$

Hence this model presents a power law distribution with an exponent $3 / 2$ for the size distribution of clusters, with an exponential cut-off. When $p \rightarrow 1 / 2$, the exponential cut-off vanishes.

\section{SIZE DEPENDENT RATES}

We can introduce a version of the above model in which the rates are proportional to the size of a group. In this case the rate equation for the system is 


$$
\frac{d n_{s}(t)}{d t}=\frac{q}{M(t)^{2}} \sum_{r=1}^{s-1} r n_{r}(s-r) n_{s-r}-\frac{2 q s n_{s}}{M(t)}+p \delta_{s, 1} .
$$

Using a similar method to that used in the previous section, but with an alternative generating function, it is simple to show that for large $s$

$$
c_{s} \sim\left(\frac{p}{1-p}\right)^{s} s^{-5 / 2}
$$

Thus, although we do not find an exact analytical solution for this variant of the model, we find that large groups are approximately power law distributed with an exponential cut-off with an exponent $5 / 2$. As $p \rightarrow 1 / 2$, the exponential cut-off vanishes.

\section{DISCUSSION}

We have discussed various different kinetic models of herding [11-13] and have introduced an alternative kinetic model which incorporates both growth and coagulation. The system grows as new agents are introduced and coagulation allows groups of agents to be formed. This is in contrast to the models proposed in Refs. [8,11], where there are a fixed number of agents.

We have presented an exact solution to this model and found that it exhibits a power law distribution with an exponential cut-off for the size distribution of clusters and hence for the distribution of returns. The model is not self-organised as the parameter $p$ has to be close to $1 / 2$ to ensure the appearance of groups of all sizes and only when $p$ is close to this value, the system displays a power law distribution for large groups where the exponent is $3 / 2$.

We have also considered a variant of the model where rates are proportional to the size of a group. Although we do not obtain an exact solution, we find that for large $s$ only, the system displays a power law distribution with an exponential cut-off. In this case, when $p \rightarrow 1 / 2$, the system gives a power law distribution for large groups but with an exponent $5 / 2$. 
These results agree to those found in Refs. $[7,8,11]$ where the models exhibit a power law distribution of returns with an exponential cut-off and in order to obtain a power law, the parameters require tuning. However, other models $[12,13]$ exhibit a power law distribution of returns for all its parameter values and the exponent of the power law distribution, in each model, can be varied continuously as it is dependent on the parameter $p$. Hence, whilst some models have a power law distribution of group sizes with a tuneable exponent, others have exponents that are determined by the model as universal exponents.

In conclusion, there seems to be a number of different processes that can give a power law distribution for group sizes. It seems as though the precise details of the processes are unimportant and the essential, universal, important ingredient for these models is the mean-field, long ranged interaction nature of all these systems. This can be related to the modern, highly connected world we live in, where social interactions are also considered to be mean-field and non-local in character. Models of this type can be expected to mimic simple processes in social networks, financial and economic phenomena where individuals share information by forming groups and break up when the information has been used. Processes for which this approach can be used range from information or rumour spreading, particularly in financial markets, through the take up of the latest craze, such as video phones or The Lord of the Rings, to epidemiological studies of disease and epidemic spread. 


\section{REFERENCES}

[1] B. Mandelbrot, J. Business 36 (1963) 394.

[2] R.N. Mantegna, H.E. Stanley, An Introduction to Econophysics: Correlations and Complexity in Finance, Cambridge University Press, Cambridge, 2000.

[3] L.A.N. Amaral, V. Plerou, P. Gopikrishnan, M. Meyer, H.E. Stanley, Int. J. Theor. Appl. Finance 3 (2000) 365.

[4] O.V. Pictet, M. Dacorogna, U.A. Muller, R.B. Olsen, J.R. Ward, J. Banking and Finance 14 (1997) 1189.

[5] S. Bikhchandani, S. Sharma, IMF Staff Papers 47 (2001) 279.

[6] K. Kim, S.-M. Yoon, Y. Kim, Herd behavior of returns in the futures exchange market (cond-mat/0304143).

[7] R. Cont, J.-P. Bouchaud, Macroeconomic Dynamics 4 (2000) 170.

[8] V.M. Eguíluz, M.G. Zimmermann, Phys. Rev. Lett. 85 (2000) 5659.

[9] R. D’Hulst, G.J. Rodgers, Physica A 280 (2000) 554.

[10] R. D’Hulst, G.J. Rodgers, Eur. Phys. J. B 20 (2001) 619.

[11] R. D’Hulst, G.J. Rodgers, Int. J. Theor. Appl. Finance 3 (2000) 609.

[12] G.J. Rodgers, D. Zheng, Physica A 308 (2002) 375.

[13] G.J. Rodgers, Y.J. Yap, Eur. Phys. J. B 28 (2002) 129.

[14] B. Rosenberg, J.A. Ohlson, J. Fin. Quant. Analysis 11 (1976) 393. 